\title{
ARTICLE OPEN \\ Rapid diagnostic assay for detection of cellulose in urine as biomarker for biofilm-related urinary tract infections
}

\author{
Haris Antypas (D) ${ }^{1}$, Ferdinand X. Choong ${ }^{1}$, Ben Libberton ${ }^{1,4}$, Annelie Brauner ${ }^{2,3}$ and Agneta Richter-Dahlfors (D)
}

The ability of uropathogenic Escherichia coli (UPEC) to adopt a biofilm lifestyle in the urinary tract is suggested as one cause of recurrent urinary tract infections (UTIs). A clinical role of UPEC biofilm is further supported by the presence of bacterial aggregates in urine of UTI patients. Yet, no diagnostics exist to differentiate between the planktonic and biofilm lifestyle of bacteria. Here, we developed a rapid diagnostic assay for biofilm-related UTI, based on the detection of cellulose in urine. Cellulose, a component of biofilm extracellular matrix, is detected by a luminescent-conjugated oligothiophene, which emits a conformation-dependent fluorescence spectrum when bound to a target molecule. We first defined the cellulose-specific spectral signature in the extracellular matrix of UPEC biofilm colonies, and used these settings to detect cellulose in urine. To translate this optotracing assay for clinical use, we composed a workflow that enabled rapid isolation of urine sediment and screening for the presence of UPECderived cellulose in $<45 \mathrm{~min}$. Using multivariate analysis, we analyzed spectral information obtained between 464 and $508 \mathrm{~nm}$ by optotracing of urine from 182 UTI patients and 8 healthy volunteers. Cellulose was detected in $14.8 \%$ of UTI urine samples. Using cellulose as a biomarker for biofilm-related UTI, our data provide direct evidence that UPEC forms biofilm in the urinary tract. Clinical implementation of this rapid, non-invasive and user-friendly optotracing diagnostic assay will potentially aid clinicians in the design of effective antibiotic treatment.

npj Biofilms and Microbiomes (2018)4:26; doi:10.1038/s41522-018-0069-y

\section{INTRODUCTION}

Biofilms are linked with chronic and recurrent infections that are resistant to treatments and hard to eradicate. ${ }^{1}$ In biofilm-related infections, the microbial community can be found directly associated with a patient's tissue or with foreign bodies, such as medical devices or implants. Biofilms are defined by the presence of bacterial aggregates embedded in a self-produced extracellular matrix (ECM) composed of extracellular polymeric substances (EPS). ${ }^{2}$ The tolerance to antibiotic treatment is mainly attributed to the distinct physiology bacteria adopt within a biofilm, but also to the specific microenvironment of the infection site. ${ }^{3}$ The metabolic heterogeneity of bacteria and the complex structure of a biofilm, combined with the hypoxic environment at the infection site, often accounts for the ineffectiveness of otherwise clinically relevant antibiotic treatments. ${ }^{4-10}$

Patients with urinary tract infection (UTI) often suffer from recurrent infections, ${ }^{11}$ which may be attributed to biofilms. ${ }^{11-13}$ Uropathogenic Escherichia coli (UPEC), the major causative agent of UTI, produces biofilm in vitro with the polysaccharide cellulose and amyloid protein curli as the major EPS. ${ }^{14,15}$ Studies in animal models have shown that UPEC most likely forms biofilm to facilitate colonization of the renal proximal tubule. ${ }^{16}$ Intracellular bacterial communities forming biofilm-like aggregates have been observed on the prostate glands, ${ }^{17}$ within the superficial cells of the bladder ${ }^{18}$ as well as in urine from UTI patients. ${ }^{19,20}$ Despite this evidence, no clinically established methods exist that provide a definitive diagnosis of biofilm-related UTI.
In routine clinical diagnostics of UTI, culture-dependent testing is based on planktonically growing cultures. While this approach is well suited to identify bacterial species, it fails to define whether bacteria originally formed biofilm in the patient. Similarly, genotypic methods cannot discriminate between planktonic bacteria and a biofilm lifestyle. ${ }^{21-23}$ To date, microscopy-based methods are commonly used to detect bacterial aggregates directly in the patient samples. These aggregates, assumed to represent a biofilm, are visualized by non-specific dyes, such as the Gram stain, or by species-specific staining, exemplified by fluorescence in situ hybridization (FISH) ${ }^{24}$ and peptide nucleic acid FISH (PNA FISH), using fluorescence confocal microscopy. ${ }^{25}$ To visualize EPS, carbohydrate stains such as alcian blue, calcofluor, and ruthenium red are applied along with fluorescently labeled lectins. $^{26-29}$ The interpretation of such analysis is, however, subjective as these stains are far from specific for biofilm components. In a limited number of cases, biofilm-specific EPS detection in patient samples has been achieved. Immunofluorescence microscopy using antibodies specific for the EPS alginate was used to detect Pseudomonas aeruginosa biofilms ${ }^{24}$ and immuno-electron microscopy using antibodies targeting the amyloid curli protein was used to detect UPEC biofilms. ${ }^{15}$ Despite specific reporting of biofilms, these advanced and labor-intensive techniques are of limited use for routine analysis in a clinical laboratory, where hundreds of samples can arrive daily.

We recently reported optotracing as a new method for realtime, in situ detection and differentiation of the Salmonella biofilm components curli and cellulose. ${ }^{30}$ Optotracing is based on a class

\footnotetext{
${ }^{1}$ Department of Neuroscience, Swedish Medical Nanoscience Center, Karolinska Institutet, Stockholm, Sweden; ${ }^{2}$ Department of Microbiology, Tumor and Cell Biology, Karolinska Institutet, Stockholm, Sweden and ${ }^{3}$ Division of Clinical Microbiology, Karolinska University Hospital, Stockholm, Sweden

Correspondence: Agneta Richter-Dahlfors (Agneta.Richter.Dahlfors@ki.se)

${ }^{4}$ Present address: MAX IV Laboratory, Lund University, Lund, Sweden
}

Received: 20 June 2018 Revised: 29 September 2018 Accepted: 9 October 2018

Published online: 26 October 2018 
Table 1. Characterization of urine from healthy volunteers

\begin{tabular}{lll}
\hline Sample \# & Urine culture & \\
\cline { 2 - 3 } & Bacterial load (CFU/ml) & Organism \\
\hline 1 & $1 \times 10^{2}$ & Enterococcus spp. \\
2 & 0 & $\mathrm{NA}^{\text {a }}$ \\
3 & $2 \times 10^{3}$ & Lactobacillus \\
4 & $3 \times 10^{2}$ & Staphylococcus or Streptococcus \\
5 & $2 \times 10^{2}$ & Staphylococcus or Streptococcus \\
6 & $4 \times 10^{2}$ & Staphylococcus or Streptococcus \\
7 & $1 \times 10^{2}$ & Enterococcus spp. \\
8 & $1 \times 10^{2}$ & Enterococcus spp. \\
\hline aNot applicable & \\
\hline
\end{tabular}

of non-toxic molecules, the luminescent-conjugated oligothiophenes (LCOs), which are flexible conjugated polymers emitting conformation-dependent fluorescence spectra. ${ }^{31}$ Binding of the heptameric LCO heptamer formyl thiophene acetic acid (h-FTAA) to EPS molecules in Salmonella biofilm resulted in linearization of the oligothiophene backbone of h-FTAA, which was observed as a red shift of the excitation spectrum compared to that from unbound h-FTAA. The spectral signature from h-FTAA bound to cellulose shows unique peaks at 464 and $488 \mathrm{~nm}$, which are characteristic of cellulose interaction. A detailed analysis of the molecular interaction between h-FTAA and the cellulose polysaccharide showed an exceptional specificity to $\beta-1,4$ configured glucans. ${ }^{32}$ This suggests a universal use of this optotracer for the detection of cellulose across biological kingdoms.

Cellulose production by UPEC serves as an indication that bacteria have entered the biofilm mode of growth. ${ }^{33-36}$ As cellulose is not naturally found within the human body, we hypothesized that any cellulose identifiable in the urine of UTI patients must have been expelled from UPEC biofilms in the urinary tract. Here, we investigated whether biofilm is formed in UTI patients by developing an assay for detection of cellulose in urine, and whether cellulose can be used as a candidate diagnostic biomarker for biofilm-related UTIs.

\section{RESULTS}

Evaluation of cellulose optotracing directly in urine

Human urine represents a complex aqueous solution of metabolites with significant native fluorescence. ${ }^{37}$ Since this may interfere with optotracing, we started by analyzing urine's fluorescence in the spectral range relevant for cellulose detection. To this end, we collected urine from eight healthy volunteers. Dipstick testing in these samples showed negative results for all UTI-associated parameters and urine cultures verified the absence of UPEC. Other bacterial species found were well below the limit for bacteriuria (Table 1). Following transfer of urine aliquots into 96-well plates, a plate reader was used to record excitation spectra of each sample at $300-520 \mathrm{~nm}$ with emission collected at $545 \mathrm{~nm}$. Phosphatebuffered saline (PBS) was profiled alongside urine samples for comparison. Analysis of data in a spectral plot (spec-plot) revealed substantial native fluorescence across the full range of excitation wavelengths (Ex. $\lambda$ ) (Fig. 1a). Individual variations were observed, with maximal relative fluorescence units ( $\left.\mathrm{RFU}_{\text {max }}\right)$ ranging between 9 and $31 \times 10^{3}$. We further analyzed the data in a normalized spec-plot, by assigning $0 \%$ to the lowest and $100 \%$ to the highest RFU in each sample. This showed that the wavelengths corresponding to the maximum emitted fluorescence $\left(\right.$ Ex. $\lambda_{\text {max }}$ ) were between 360 and $380 \mathrm{~nm}$ in all urine samples (Fig. 1b). In contrast to urine, PBS showed $\mathrm{RFU}_{\max }$ as low
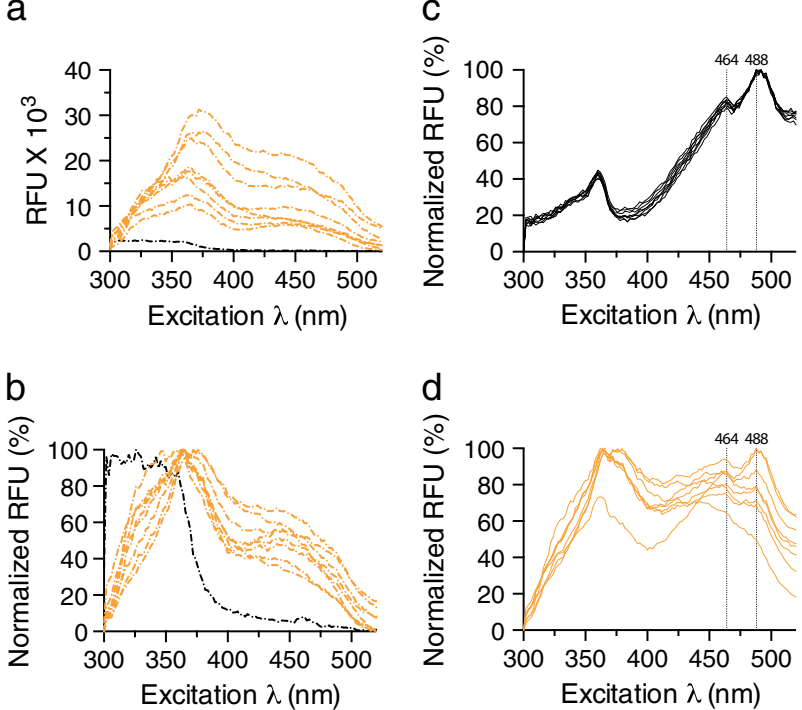

Fig. 1 Optotracing for direct detection of cellulose in urine. a Excitation spectra and $\mathbf{b}$ normalized excitation spectra of urine from eight healthy volunteers (dash-dot orange) and PBS (dash-dot black) excited at $300-520 \mathrm{~nm}$ with emission collected at $545 \mathrm{~nm}$. Average fluorescence of two technical replicates for each sample is shown. c, d Normalized excitation spectra showing optotracing of $\mathrm{M}$. cellulose mixed with h-FTAA in c PBS and $\mathbf{d}$ healthy urine. Each line represents the average normalized fluorescence from three technical replicates per preparation. Dotted vertical lines $=464$ and $488 \mathrm{~nm}$ representing the cellulose signature

as $2.5 \times 10^{3}$, and this signal decreased even more at wavelengths longer than $380 \mathrm{~nm}$ (Fig. 1a, b). Overall, urine was predominantly fluorescent at the shorter wavelengths, which are of lesser importance during cellulose optotracing, as the cellulose spectral signature is expected at wavelengths longer than $400 \mathrm{~nm} .{ }^{30}$ We concluded that cellulose optotracing ought to generate signals with enough fluorescent amplitude to be visible at wavelengths longer than $400 \mathrm{~nm}$, despite urine's native fluorescence.

Next, we identified the optical signatures of cellulose by performing optotracing with h-FTAA in eight preparations of $125 \mu \mathrm{g} / \mathrm{ml}$ microcrystalline cellulose (M. cellulose) in PBS. The normalized spec-plots showed great consistency as spectra from the eight preparations were superimposed on each other. Two distinct peaks were observed, one with Ex. $\lambda_{\max }$ at $488 \mathrm{~nm}$ and one with lower RFU at $464 \mathrm{~nm}$ (Fig. 1c). These two peaks define the cellulose optical signature and they are clear indicators of cellulose in the sample. ${ }^{30}$ To define the detection limit of our method, we made 2-fold dilution series of the $M$. cellulose preparations in PBS, and included PBS-only as blank. When performing optotracing, we obtained a clear spectral signature in all of the eight cellulose preparations containing $62.5 \mu \mathrm{g} / \mathrm{ml}$ (Fig. $\mathrm{S} 1 \mathrm{a})$. A majority of the samples, five out of eight preparations, also provided clear cellulose signatures at $31.3 \mu \mathrm{g} / \mathrm{ml}$ (Fig. S1b). In contrast, the cellulose signal was absent at lower concentrations (Fig. S1c, d), with some of the spectra mimicking the PBS blank (Fig. S1e). We thus conclude that the detection limit of optotracing for cellulose in PBS ranges between 31.3 and $62.5 \mu \mathrm{g} / \mathrm{ml}$.

To test whether optotracing for cellulose also can be achieved in urine, we spiked the eight urine samples from healthy volunteers with $125 \mu \mathrm{g} / \mathrm{ml}$ each of $M$. cellulose, added h-FTAA, and collected the excitation spectra. We detected the cellulose signature in two of the samples, with Ex. $\lambda_{\max }$ at $488 \mathrm{~nm}$ and the minor peak at $464 \mathrm{~nm}$ (Fig. 1d). In the remaining samples, we observed only weak cellulose spectral signatures or none at all, suggesting that the cellulose optotracing signal is dominated by the emission of urine's native fluorescence. To define the 
detection limit, we spiked the eight healthy urine samples with the $M$. cellulose, made 2-fold serial dilutions of each sample, and performed optotracing, using urine-only samples as blank. A clear cellulose signature was observed in two out of eight samples at $62.5 \mu \mathrm{g} / \mathrm{ml}$ (Fig. S1f), while spectra at all lower concentrations resembled samples containing urine only (Fig. S1g-j). Whereas these results demonstrate that cellulose optotracing directly in urine is feasible, native fluorescence is indeed a concern for the consistency of the detection limit, especially since we noted a great variability in the intrinsic fluorescence between individuals. Samples with high fluorescence are thus at risk of affecting optotracing's detection sensitivity. Collectively, these results show that PBS is better suited for cellulose optotracing than urine, as consistent results are generated at a lower detection limit.

\section{Detection of cellulose in UPEC biofilms}

We next determined if cellulose can be detected in its native complexity within UPEC biofilms by optotracing. We used UPEC No. 12, a clinical uropathogenic $E$. coli strain isolated from a child with pyelonephritis. ${ }^{15}$ This wild-type (WT) strain is known to form biofilm when grown on agar plates under osmotic stress at $37^{\circ} \mathrm{C}$. In the presence of Congo red, the characteristic colony morphology termed rdar (red, dry, and rough) forms, indicating the presence of cellulose and curli in the biofilm's ECM (Fig. 2a, WT). The isogenic strain UPEC No. $12 \triangle b c s A$, which has a mutation in the gene encoding the cellulose synthase catalytic subunit, renders this strain unable to produce cellulose. This is manifested by an inability to form the rdar morphotype (Fig. 2a, $\Delta b c s A$ ). To perform optotracing for cellulose in UPEC biofilms, we harvested biofilm colonies from WT and $\triangle b c s A$ strains, resuspended them in PBS containing h-FTAA, and recorded the excitation spectra. Optotracing of the WT showed two peaks in the normalized specplot, both red-shifted compared to unbound h-FTAA (Fig. 2b). The appearance of Ex. $\lambda_{\max }$ at $464 \mathrm{~nm}$ and a minor peak at $488 \mathrm{~nm}$, compared to Ex. $\lambda_{\max }$ at $400 \mathrm{~nm}$ of the unbound h-FTAA, strongly indicates the presence of cellulose. The spectrum of the mutant $\triangle b c s A$ lacked both peaks, which confirms the absence of cellulose in the sample, as expected from the genotype of this strain. The Ex. $\lambda_{\max }$ at $450 \mathrm{~nm}$ of the mutant strain results from binding of the optotracer to unknown components in the complex ECM.

To investigate whether we can detect cellulose from UPEC biofilm in urine, we spiked one aliquot of healthy urine containing h-FTAA with biofilm from the WT strain, and another with biofilm from the mutant $\triangle b c s A$. As negative control, we used urine to which only h-FTAA was added. Analysis of the normalized specplot showed little differences between spiked and unspiked urine. Signals from the native fluorescence of urine dominated the three spectra, shown by Ex. $\lambda_{\max }$ at $360-380 \mathrm{~nm}$ (Fig. 2c). A marginally higher fluorescence in the range $425-500 \mathrm{~nm}$ was observed in urine spiked with biofilm from WT bacteria compared to biofilm from the mutant $\Delta b c s A$ and the negative control. Signals were thus not sufficient to consitute a cellulose signature.

To improve the sensitivity of optotracing for cellulose in native biofilms, we developed an alternative workflow. Knowing that optotracing generates very consistent signals for $M$. cellulose in PBS (Fig. 1c), we transferred bacteria and their biofilm components from urine to PBS before optotracing. A small volume of urine $(1 \mathrm{ml})$ spiked with biofilm from the WT or the mutant $\triangle b c s A$, as well as urine only (negative control), were centrifuged for $15 \mathrm{~min}$. Pellets were resuspended in $0.5 \mathrm{ml}$ PBS containing h-FTAA and then optotracing for cellulose was performed. The normalized spec-plot showed a distinctly red-shifted spectrum for the WT compared to the negative control, the cellulose-specific Ex. $\lambda_{\max }$ at $464 \mathrm{~nm}$ and the accessory peak at $488 \mathrm{~nm}$ (Fig. 2d). In contrast, biofilm from the mutant $\triangle b c s A$ showed Ex. $\lambda_{\max }$ at $450 \mathrm{~nm}$ with no indications of cellulose in the sample. Collectively, this shows that a

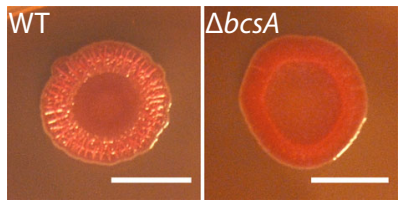

b

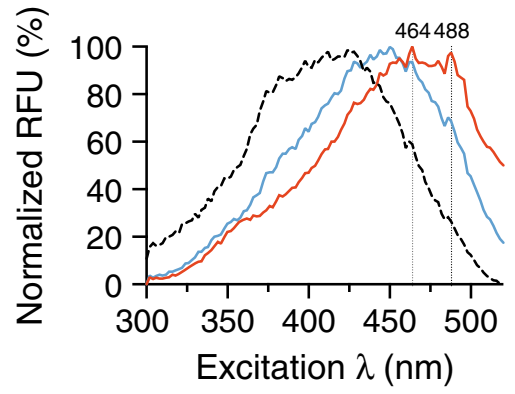

C
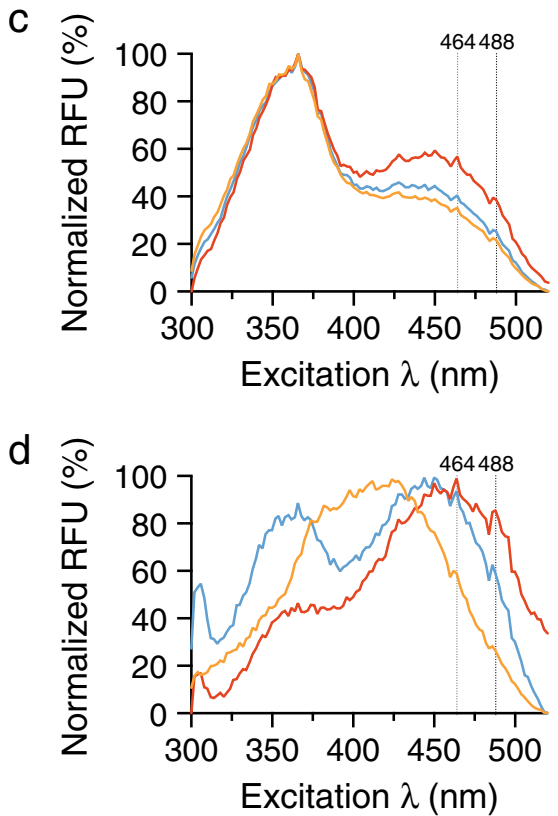

Fig. 2 Cellulose detection in UPEC biofilms by optotracing. a Colony morphotypes of UPEC No. 12 WT and $\triangle b C S A$ on Congo red agar plates. Scale bar $=1 \mathrm{~cm}$. b Normalized spec-plot of biofilms from UPEC No. 12 WT (red) and $\triangle b c s A$ (blue) resuspended in PBS containing h-FTAA, and of unbound h-FTAA in PBS (black). Average normalized fluorescence from three biological replicates is shown. $c$ Normalized spec-plot of biofilms from UPEC No. 12 WT (red) and $\triangle b c s A$ (blue) resuspended in urine containing $\mathrm{h}-\mathrm{FTAA}$. Urine containing only h-FTAA (orange) was included for comparison. Average normalized fluorescence from three technical replicates from one representative experiment is shown. d Normalized specplot showing optotracing of samples presented in c after they were centrifuged at $15,700 \times g$ and resuspended in $500 \mu \mathrm{l} \mathrm{PBS}$. Average normalized fluorescence from three technical replicates from one representative experiment is shown. Dotted vertical lines $=464$ and $488 \mathrm{~nm}$ representing the cellulose signature

the transfer of biofilm from urine to PBS increases sensitivity for cellulose detection by optotracing.

Cellulose as a biomarker for biofilm-associated UTIs

Despite evidence of biofilm-like bacterial aggregates in urine from UTI patients, biofilm EPS, such as cellulose, have never been detected. Based on our rapid and user-friendly workflow for urine analysis, we investigated whether optotracing could be applied to 
detect cellulose in clinical urine samples. Urine samples from 182 patients, clinically diagnosed with UTI caused by UPEC, were collected. For comparative purposes, we included urine from the eight healthy volunteers, as well as biofilm samples from UPEC No. $12 \mathrm{WT}$ and the mutant $\triangle b c s A$, which served as cellulose-positive (WT) and cellulose-negative $(\triangle b c s A)$ references in the analysis. We isolated the sediments from all urine samples and resuspended them in PBS. By performing optotracing, we obtained spectral information from all 192 samples. To enable unbiased analysis of the spectral data and easy comparison of the multiple fine spectral details represented by the varying fluorescence intensity at each nanometer, we used principal component analysis (PCA). This type of analysis was applied to spectral information obtained between 464 and $508 \mathrm{~nm}$, where the cellulose optical signature is expected. Principal component 1 (PC1) and principal component 2 (PC2) were selected, as they were found to account for $>99 \%$ of the variation across excitation spectra from all strains. We applied $k$ means clustering to organize the spread of samples across PC1 and $P C 2$, and the Elbow method to define the optimal number of clusters present in our PCA. Based on the similarities among excitation spectra, this analysis grouped samples into three clusters, which we colored red, gray, and blue in the PCA plot (Fig. 3).

The red cluster at the top right of the PCA plot included 27 UTI samples and the cellulose-positive reference (WT) (Fig. 3). Since this suggested the presence of cellulose, we selected representative UTI samples based on their distribution across this cluster for further analysis. UTI samples located to the top right (see nos. 66, $87,146,73$ in the magnified red cluster in Figure S2a) showed Ex. $\lambda_{\max }$ at $488 \mathrm{~nm}$ and a smaller peak at $464 \mathrm{~nm}$ (Fig. S2b). This pattern overlapped with the WT strain, and resembled the typical signature of $M$. cellulose (Fig. 1c), suggesting a substantial abundance of cellulose in these samples. UTI samples spreading

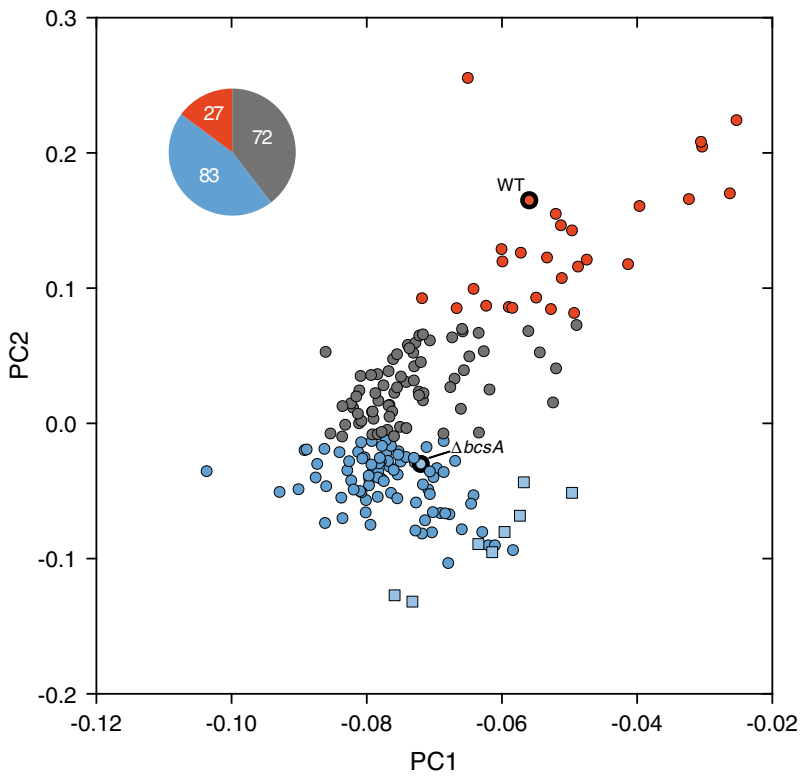

Fig. 3 Optotracing of cellulose in urine from UTI patients. Principal component analysis (PCA) plot of cellulose optotracing in 182 urine samples from UTI patients (circle), eight healthy urine samples (square) and two biofilm preparations from UPEC No. 12 WT (thick border red circle) and $\triangle b c s A$ (thick border blue circle). Data from normalized excitation spectra in the cellulose signature wavelength range of 464-508 nm were analyzed with PCA and clustered with $k$ means clustering. The three generated clusters differentiate samples that are positive (red) and negative (blue) for cellulose, as well as samples with insufficient discriminatory performance (gray). The pie chart inset shows the number of UTI samples in each of the three clusters towards the bottom left (nos. 18, 65, 28, 17) showed Ex. $\lambda_{\max }$ at $464 \mathrm{~nm}$, and an accompanying smaller peak at $488 \mathrm{~nm}$ (Fig. S2b). Overall, these data showed that cellulose is present in UTI samples. PCA and $k$-means clustering on defined and unknown samples enabled us to rapidly and accurately identify those containing cellulose. The altered position of Ex. $\lambda_{\max }$ between 464 and $488 \mathrm{~nm}$ observed in the spectra of this cluster may reflect differences in the amount of cellulose and the biofilm complexity in each sample.

The blue cluster at the lower end of the PCA plot includes 83 UTI samples and the cellulose-negative reference $(\triangle b c s A)$, (Fig. 3). As this suggested that these samples lacked cellulose, we selected representative samples across the full distribution of the blue cluster for further evaluation (Fig. S3a). Indeed, the normalized spec-plots from each selected UTI sample were similar with the cellulose-negative reference sample (Fig. S3b). This suggested that a majority of urine samples in our UTI collection does not contain cellulose.

The urine samples from our eight healthy volunteers were also located in the blue cluster, albeit relatively isolated in the periphery of the cluster (Fig. 3 and S3a). The normalized specplots of these samples (nos. 1-8) showed no presence of cellulose (Fig. S3c). Their distinct position at the periphery of the cluster showed that the content of healthy urine markedly differs from that of samples from patients with UTI. Thus, optotracing seems to differentiate healthy from infected urine.

The gray cluster in the central part of the PCA plot contains 72 UTI samples (Fig. 3). Close inspection of this cluster in a magnified plot showed an even sample distribution in the area between the red and blue cluster (Fig. S4a). The normalized spec-plot of samples closest to the red cluster (nos. 23, 57, 38) showed Ex. $\lambda_{\max }$ at $464 \mathrm{~nm}$ and a low-amplitude spike at $488 \mathrm{~nm}$ (Fig. S4b), suggesting the presence of cellulose. Analysis of samples located in the central area of the gray cluster (nos. 134, 69, 68) showed red-shifted Ex. $\lambda_{\max }$ compared to the cellulose-negative control $\triangle b c s A$. However, lack of cellulose-specific peaks at 464 and $488 \mathrm{~nm}$ suggests an absence of cellulose in these samples. Finally, analysis of samples located close to the blue cluster (nos. 85, 110) showed Ex. $\lambda_{\max }$ at wavelengths shorter than $450 \mathrm{~nm}$ (Fig. S4b). The lack of peaks in the cellulose-specific region in most of the gray cluster samples suggests the absence of cellulose. Yet, these spectra differed from that of the cellulose-negative reference. We thus concluded that the discriminatory performance of the spectra generated from samples in the gray cluster was insufficient to determine the presence or absence of cellulose.

Taken together, our results support the application of optotracing combined with PCA for rapid screening and detection of cellulose in urine samples, as a biomarker for biofilm-related UTI. This analysis showed that cellulose-based biofilms are present in $14.8 \%$ of urine samples from UTI patients.

\section{DISCUSSION}

Biofilm is increasingly recognized as a main virulence factor in chronic and recurrent infections. ${ }^{38}$ Despite the progress made in understanding biofilm's role in infection, development of diagnostic methods for biofilm presence is lagging behind. This is primarily due to lack of tools that detect biofilm-specific components. Here, we have developed a culture-independent assay that rapidly detects cellulose as a UPEC biofilm marker in urine from UTI patients. A urine sample of $1 \mathrm{ml}$ is sufficient to isolate bacterial components and screen them for the presence of cellulose using the optotracing technology. Testing is based on standard instrumentation available in a clinical microbiology laboratory, and it is easily performed with a total hands-on time of less than an hour. To meet the clinical demand for rapid and high-throughput data analysis, we applied PCA and $k$-means clustering as a powerful, non-biased way to process results for 
interpretation. In this study, cellulose optotracing of 182 urine samples from patients with UTI indicated that cellulose was present in $14.8 \%$ of the patients. This proof of concept demonstrates that cellulose optotracing has great potential as a diagnostic method for biofilm-related infections.

Existing methods used for direct detection of biofilm in UTI samples are non-specific as they are based on detecting bacterial aggregates under microscopy, rather than specific EPS produced when bacteria enter a biofilm lifestyle. ${ }^{24-29}$ Interpretation of such results is subjective and does not provide a definitive way of diagnosing an infection as biofilm-associated. The core theory of the optotracing methodology applied here is the detection of a specified polysaccharide within a sample, based on a unique spectral signature. In this study, cellulose was the polysaccharide in focus as it is a major component of the ECM in UPEC biofilms. Owing to the ability of optotracing to specifically detect cellulose, we were able to determine whether a UTI was biofilm-associated.

Since the optotracer molecules bind directly to the polysaccharide backbone of cellulose, they can potentially be applied as universal detectors for cellulose produced by microbes since detection is independent of species-specific differences in cellulose morphology, density, and crystallinity. ${ }^{32}$ Furthermore, diagnostic optotracing of urine samples can be completed in less than an hour, owing to the simple sample preparation and the ability of optotracing to instantaneously generate spectral signatures upon cellulose binding.

Optotracing is a pioneering diagnostic assay for detection of native cellulose in urine. Central to its implementation in complex clinical samples is the non-disruptive nature of the method. All established methods involve hydrolysis of cellulose and detection of glucose, the monomeric unit of cellulose. Such methods are not suitable for cellulose diagnostics, as glucose in urine from UTI patients can originate from multiple host and bacterial sources. This made it difficult to establish a reference method to define the diagnostic accuracy of optotracing. We took, however, several measures to reduce the risk of misdiagnosing cellulose in samples. We used PBS as assay matrix to ensure low native fluorescence, increase the consistency of results during optotracing, and achieve a lower detection limit for cellulose. This minimized the risk of false-negative results due to the variability of urine among individuals. To increase the likelihood of detecting cellulose, we concentrated each sample by pelleting bacterial components. However, this also concentrated host components indiscriminately, such as red and white blood cells or exfoliated epithelial cells, into collected pellets. Although it is currently unknown whether host components would compromise optotracing, future improvements in sample pre-processing may increase detection sensitivity. It is also important to note that this method is specific for cellulose-expressing biofilms; infections caused by celluloseexpressing microbes other than UPEC would also give a positive result. Similarly, a negative result would not exclude a biofilm infection, as the EPS constituents may differ between strains. The risk of obtaining false-positive results is low. Cellulose is not naturally produced by humans, so its presence in urine ought to be of microbial origin. Bacteria other than UPEC found in low amounts in healthy urine samples (Enterococcus, Lactobacillus, Staphylococcus, Streptococcus spp.) did not generate any cellulose spectral signature. Among the species known to produce cellulose as a component of their biofilm ECM, only UPEC is of high clinical relevance for UTIs. ${ }^{39}$

We believe that a method for cellulose-based biofilm diagnostics would be of great clinical relevance. Information on biofilm diagnostics combined with species identification and antimicrobial susceptibility testing results from planktonic cultures could aid in the better design of antibiotic regimens. Although we are still far from establishing clinical breakpoints for biofilm-related infections, there is reported evidence for the differential effectiveness of antibiotics on biofilms. Some antibiotics have been shown to preferentially kill metabolically active bacteria in the outer layers of the biofilm, while others kill metabolically inactive bacteria in its inner part. ${ }^{40-43}$ The low oxygen levels in biofilms is another factor that affects the activity of antibiotics, such as Ciprofloxacin. ${ }^{44}$ Moreover, the biofilm matrix can trap enzymes, such as $\beta$-lactamases that hydrolyze $\beta$-lactam antibiotics. ${ }^{45}$ This means that the dose and the duration of therapy may have to be adjusted in the presence of biofilm and $\beta$-lactamase stable antibiotics, such as meropenem or imipenem, could be used instead. By knowing the presence of biofilm in a patient, the clinician could select a more effective panel of antibiotics and also attempt a combinational or sequential antibiotic therapy. ${ }^{3}$ Biofilm diagnostics could also help to evaluate alternative therapies to target biofilm. ${ }^{38,46}$ Finally, by establishing biofilm diagnostics in the clinics, a surveillance system can be implemented for patients with chronic and recurrent infections to evaluate the success of antibiotic treatment, or for catheterized patients at risk of developing catheter-associated infections.

In conclusion, the development and clinical testing of our method for cellulose diagnostics in biofilm UTI shows the potential of optotracing in clinical diagnostics. As the technology matures, we foresee an expansion of optotracing to polysaccharides and functional amyloids in ECM of other clinically significant microbial biofilms. When combined with multivariate analysis, optotracing showed promising discriminatory performance between healthy and infected urine samples. The relevance of this finding for clinical diagnostics remains to be seen. Ultimately, we envision optotracing of EPS as an easily integrable method alongside other routine diagnostics in the clinical laboratory that would improve diagnosis and treatment of biofilm infections.

\section{METHODS}

\section{Study design}

Research was conducted in full conformance with the laws and regulation of Sweden. Collection and analysis of anonymous UTI urine samples was approved by Karolinska Institutet, Karolinska University Hospital and by Regionala etikprövningsnämnden (EPN), Stockholm (The Regional Ethics Committee, Stockholm), decision reference 2017/448-31/1. Since urine samples were collected anonymously, this study was exempted from the requirement of a written informed consent.

\section{Urine collection and characterization}

Midstream urine from eight anonymous healthy volunteers was collected and immediately screened with a urine dipstick (Multistix ${ }^{\circledR} 10$ SG reagent strip, Siemens, Germany). Urine cultures were performed by streaking $10 \mu \mathrm{l}$ urine on Brilliance ${ }^{\mathrm{TM}}$ UTI Agar (Oxoid, UK), and incubating the plates at $37^{\circ}$ C. Next day, colony-forming units (CFUs) were counted, and bacterial species were identified based on the colony color. To investigate urine's native fluorescence, $2 \times 100 \mu \mathrm{l}$ from each healthy urine sample were transferred in a transparent, flat-bottom 96-well plate, and excitation spectra of $300-520 \mathrm{~nm}$ with emission collected at $545 \mathrm{~nm}$ were collected using an Infinite ${ }^{\circledR}$ M1000 PRO plate reader (TECAN, Mannedorf, Switzerland).

Aliquots of $3-5 \mathrm{ml}$ of urine samples were collected anonymously from 182 patients with confirmed UTI caused by $>10^{5} \mathrm{CFU} / \mathrm{ml}$ of UPEC from Karolinska University Hospital, Stockholm, Sweden. All samples were immediately analyzed, and discarded after use.

\section{Biofilm cultivation and Congo red morphotyping}

The clinical strain UPEC No. 12 (WT), isolated from a patient with UTI, and the mutant strain WE1 $b c s A:: C \mathrm{Cm}(\triangle b c s A)$ were used as an isogenic pair of strains for controlled expression of cellulose. ${ }^{15}$ Single colonies of each strain were grown overnight in LB broth at $37^{\circ} \mathrm{C}$. The overnight cultures were washed twice in LB broth without sodium chloride (LB n.s.) and pellets were resuspended again in LB n.s. To grow biofilm colonies, $10 \mu \mathrm{l}$ drops of each strain were applied on LB n.s. solid media, and plates were incubated for $48 \mathrm{~h}$ at $37^{\circ} \mathrm{C}$. For biofilm colony morphotyping, $10 \mu \mathrm{l}$ drops were applied on Congo red solid media as previously described, ${ }^{30}$ and incubated for $48 \mathrm{~h}$ at $37^{\circ} \mathrm{C}$. Pictures of all morphotypes were taken. 


\section{Cellulose optotracing}

Optotracing for cellulose was performed using the LCO h-FTAA (gift from Prof. P. Nilsson, Linköping University, Linköping, Sweden). ${ }^{47-49}$ Stock solutions $(1.5 \mathrm{mM})$ prepared in $\mathrm{dH}_{2} \mathrm{O}$ were maintained at $4{ }^{\circ} \mathrm{C}$. From each sample preparation (see below), 50-150 $\mu$ l were transferred in duplicates or triplicates to transparent, flat-bottom 96-well plates. h-FTAA was added to each well at a final concentration of $4.5 \mu \mathrm{M}$, and incubated for $15 \mathrm{~min}$ at room temperature. To collect excitation spectra, samples were excited between 300 and $520 \mathrm{~nm}$ using 2-nm steps and emission was collected at $545 \mathrm{~nm}$ in a plate reader.

To identify the cellulose detection limit, we prepared 2-fold dilution series of M. cellulose (Sigma-Aldrich, MO, USA), ranging from 125 to $7.8 \mu \mathrm{g} /$ $\mathrm{ml}$, in eight different healthy urine samples and in eight samples of PBS. We also prepared negative controls for each series with no cellulose. Samples were sonicated at 30\% amplitude using a 2-mm sonicator probe (Sonics \& Materials, CT, USA), following an on/off cycle of $5 \mathrm{~s}$ for a total of $40 \mathrm{~s}$. To prepare biofilm for optotracing, single biofilm colonies from UPEC No. 12 WT and $\triangle b c s A$ were resuspended in $500 \mu \mathrm{l}$ PBS or $1 \mathrm{ml}$ of healthy urine. To transfer bacterial components from urine to PBS before optotracing, samples were centrifuged at $15,700 \times g$ for $15 \mathrm{~min}$ at $4{ }^{\circ} \mathrm{C}$ and pellets were resuspended in fresh $500 \mu \mathrm{l}$ PBS.

To prepare the 190 urine samples for optotracing, $1 \mathrm{ml}$ of each sample was centrifuged at $15,700 \times g$ for $15 \mathrm{~min}$ at $4{ }^{\circ} \mathrm{C}$, and the urine sediment was resuspended in $500 \mu \mathrm{l}$ PBS. Resuspensions were serially diluted 1:2, 1:4, and 1:8 in PBS before optotracing. Reference samples UPEC No. 12 WT and $\triangle b c s A$ were prepared by transferring single biofilm colonies into $500 \mu \mathrm{l}$ $\mathrm{PBS}$, which were vortexed vigorously for $10 \mathrm{~s}$ followed by centrifugation at $3300 \times g$ for $15 \mathrm{~s}$, and resuspension in $500 \mu \mathrm{l}$ PBS. This washing step was repeated two more times. Washed biofilm samples were sonicated for $5 \mathrm{~s}$ at $30 \%$ amplitude on ice using a $2 \mathrm{~mm}$ sonicator probe (Sonics \& Materials, CT, USA), pelleted at $3300 \times g$ for $15 \mathrm{~s}$, and resuspended in $500 \mu \mathrm{l}$ PBS before optotracing was performed.

\section{Data analysis}

Data from excitation spectra were normalized by assigning $0 \%$ to the lowest and $100 \%$ to the maximum RFU value per individual recording. For PCA, we used the built-in function "prcomp()" in R software (version 3.2.0). The input dataset for PCA comprised of the normalized cellulose optotracing spectral data between 464 and $508 \mathrm{~nm}$ from the eight healthy and 182 infected urine samples, as well as from UPEC No. 12 WT and $\triangle b c s A$ reference samples. PC1 and PC2 were further analyzed with $k$-means clustering, by defining the number of clusters $(k)$ with the Elbow method and subsequently using the built-in function "k-means()" in R software (version 3.2.0). All plots were prepared using Prism (version 6.0).

\section{DATA AVAILABILITY}

The authors declare that data supporting the findings of this study are available upon reasonable request.

\section{ACKNOWLEDGEMENTS}

We thank Prof. P. Nilsson at the Linköping University, Sweden, for kindly providing us with the optotracer h-FTAA, as well as for providing us with scientific insight that greatly assisted our research. This work was funded by the Erling-Persson Family Foundation, the Swedish Foundation for Strategic Research, the Swedish Research Council and Carl Bennet AB (to A.R.-D.), and Stockholm County Council (ALF project), Swedish Neurological Association (Neurofonden), and grants from Karolinska Institutet (to A.B.).

\section{AUTHOR CONTRIBUTIONS}

H.A. and A.R.-D. conceived the initial hypothesis, which was further developed with contributions from F.X.C., A.B. and B.L., H.A., A.B., and A.R.-D. designed the study. H.A. performed all experiments. A.B. provided clinical guidance, collected, and prevalidated clinical samples. H.A., F.X.C., B.L., and A.R.-D. analyzed the data. H.A., F.X.C. and A.R.-D. wrote the manuscript, which was critically revised by B.L. and A.B.

\section{ADDITIONAL INFORMATION}

Supplementary information accompanies the paper on the npj Biofilms and Microbiomes website (https://doi.org/10.1038/s41522-018-0069-y).
Competing interests: H.A., F.X.C., B.L., and A.R.-D are co-inventors of patents relevant to this work. Intellectual properties are owned by Richter Life Science Development $A B$, founded by A.R.-D. F.X.C., and B.L. are shareholders of Furcifer AB. A.B. declares no competing interests.

Publisher's note: Springer Nature remains neutral with regard to jurisdictional claims in published maps and institutional affiliations.

\section{REFERENCES}

1. Costerton, J. W., Stewart, P. S. \& Greenberg, E. P. Bacterial biofilms: a common cause of persistent infections. Science 284, 1318-1322 (1999).

2. Hall-Stoodley, L. et al. Towards diagnostic guidelines for biofilm-associated infections. FEMS Immunol. Med. Microbiol. 65, 127-145 (2012).

3. Ciofu, O., Rojo-Molinero, E., Macia, M. D. \& Oliver, A. Antibiotic treatment of biofilm infections. APMIS 125, 304-319 (2017).

4. Walters, M. C. III, Roe, F., Bugnicourt, A., Franklin, M. J. \& Stewart, P. S. Contributions of antibiotic penetration, oxygen limitation, and low metabolic activity to tolerance of Pseudomonas aeruginosa biofilms to Ciprofloxacin and Tobramycin. Antimicrob. Agents Chemother. 47, 317-323 (2003).

5. Chiang, W. C. et al. Extracellular DNA shields against aminoglycosides in Pseudomonas aeruginosa biofilms. Antimicrob. Agents Chemother. 57, 2352-2361 (2013).

6. Mulcahy, H., Charron-Mazenod, L. \& Lewenza, S. Extracellular DNA chelates cations and induces antibiotic resistance in Pseudomonas aeruginosa biofilms. PLoS Pathog. 4, e1000213 (2008).

7. Bagge, N. et al. Dynamics and spatial distribution of beta-lactamase expression in Pseudomonas aeruginosa biofilms. Antimicrob. Agents Chemother. 48, 1168-1174 (2004).

8. Brooun, A., Liu, S. H. \& Lewis, K. A dose-response study of antibiotic resistance in Pseudomonas aeruginosa biofilms. Antimicrob. Agents Chemother. 44, 640-646 (2000).

9. Lewis, K. Persister Cells. Annu. Rev. Microbiol. 64, 357-372 (2010).

10. Kragh, K. N. et al. Polymorphonuclear leukocytes restrict growth of Pseudomonas aeruginosa in the lungs of cystic fibrosis patients. Infect. Immun. 82, 4477-4486 (2014).

11. Foxman, B. Recurring urinary tract infection: incidence and risk factors. Am. J. Public Health 80, 331-333 (1990).

12. Glover, M., Moreira, C. G., Sperandio, V. \& Zimmern, P. Recurrent urinary tract infections in healthy and nonpregnant women. Urol. Sci. 25, 1-8 (2014).

13. Soto, S. M. et al. Implication of biofilm formation in the persistence of urinary tract infection caused by uropathogenic Escherichia coli. Clin. Microbiol. Infect. 12, 1034-1036 (2006)

14. Ramos, N. L. et al. Characterisation of uropathogenic Escherichia coli from children with urinary tract infection in different countries. Eur. J. Clin. Microbiol. Infect. Dis. 30, 1587-1593 (2011).

15. Kai-Larsen, Y. et al. Uropathogenic Escherichia coli modulates immune responses and its curli fimbriae interact with the antimicrobial peptide LL-37. PLoS Pathog. 6, e1001010 (2010).

16. Melican, K. et al. Uropathogenic Escherichia coli $\mathrm{P}$ and Type 1 fimbriae act in synergy in a living host to facilitate renal colonization leading to nephron obstruction. PLoS Pathog. 7, e1001298 (2011).

17. Nickel, J. C. \& Costerton, J. W. Bacterial localization in antibiotic-refractory chronic bacterial prostatitis. Prostate 23, 107-114 (1993).

18. Justice, S. S. et al. Differentiation and developmental pathways of uropathogenic Escherichia coli in urinary tract pathogenesis. Proc. Natl. Acad. Sci. USA 101, 1333-1338 (2004).

19. Robino, L. et al. Intracellular bacteria in the pathogenesis of Escherichia coli urinary tract infection in children. Clin. Infect. Dis. 59, e158-e164 (2014).

20. Rosen, D. A., Hooton, T. M., Stamm, W. E., Humphrey, P. A. \& Hultgren, S. J. Detection of intracellular bacterial communities in human urinary tract infection. PLoS Med. 4, e329 (2007).

21. Choe, H. S. et al. Analysis of the distribution of bacteria within urinary catheter biofilms using four different molecular techniques. Am. J. Infect. Control 40, E249-E254 (2012).

22. Frank, D. N., Wilson, S. S., St. Amand, A. L. \& Pace, N. R. Culture-independent microbiological analysis of Foley urinary catheter biofilms. PLOS ONE 4, e7811 (2009).

23. $\mathrm{Xu}, \mathrm{Y}$. et al. Culture-dependent and -independent investigations of microbial diversity on urinary catheters. J. Clin. Microbiol. 50, 3901-3908 (2012).

24. Bjarnsholt, T. et al. Pseudomonas aeruginosa biofilms in the respiratory tract of cystic fibrosis patients. Pediatr. Pulmonol. 44, 547-558 (2009). 
25. Malic, S. et al. Detection and identification of specific bacteria in wound biofilms using peptide nucleic acid fluorescent in situ hybridization (PNA FISH). Microbiology 155, 2603-2611 (2009).

26. Hoffmann, N. et al. Novel mouse model of chronic Pseudomonas aeruginosa lung infection mimicking cystic fibrosis. Infect. Immun. 73, 2504-2514 (2005).

27. Yang, L. et al. In situ growth rates and biofilm development of Pseudomonas aeruginosa populations in chronic lung infections. J. Bacteriol. 190, 2767-2776 (2008).

28. Neu, T. R., Swerhone, G. D. W. \& Lawrence, J. R. Assessment of lectin-binding analysis for in situ detection of glycoconjugates in biofilm systems. Microbiology 147, 299-313 (2001).

29. Johnsen, A. R., Hausner, M., Schnell, A. \& Wuertz, S. Evaluation of fluorescently labeled lectins for noninvasive localization of extracellular polymeric substances in Sphingomonas biofilms. Appl. Environ. Microbiol. 66, 3487-3491 (2000).

30. Choong, F. X. et al. Real-time optotracing of curli and cellulose in live Salmonella biofilms using luminescent oligothiophenes. NPJ Biofilms Microbi. 2, 16024 (2016).

31. Klingstedt, $T$. et al. The structural basis for optimal performance of oligothiophene-based fluorescent amyloid ligands: conformational flexibility is essential for spectral assignment of a diversity of protein aggregates. Chem. Eur. J. 19, 10179-10192 (2013).

32. Choong, F. X. et al. Stereochemical identification of glucans by oligothiophenes enables cellulose anatomical mapping in plant tissues. Sci. Rep. 8, 3108 (2018).

33. Hufnagel, D. A., Depas, W. H. \& Chapman, M. R. The biology of the Escherichia coli extracellular matrix. Microbiol. Spectr. 3, 1-14 (2015).

34. DePas, W. H. et al. Iron induces bimodal population development by Escherichia coli. Proc. Natl. Acad. Sci. USA 110, 2629-2634 (2013).

35. Zogaj, X., Nimtz, M., Rohde, M., Bokranz, W. \& Römling, U. The multicellular morphotypes of Salmonella typhimurium and Escherichia coli produce cellulose as the second component of the extracellular matrix. Mol. Microbiol. 39, 1452-1463 (2001).

36. Serra, D. O., Richter, A. M. \& Hengge, R. Cellulose as an architectural element in spatially structured Escherichia coli biofilms. J. Bacteriol. 195, 5540-5554 (2013).

37. Kušnír, J., Dubayová, K., Lešková, L. \& Lajtár, M. Concentration matrices-solutions for fluorescence definition of urine. Anal. Lett. 38, 1559-1567 (2005).

38. Koo, H., Allan, R. N., Howlin, R. P., Stoodley, P. \& Hall-Stoodley, L. Targeting microbial biofilms: current and prospective therapeutic strategies. Nat. Rev. Microbiol. 15, 740-755 (2017).

39. Hay, A. D. et al. The Diagnosis of Urinary Tract infection in Young children (DUTY): a diagnostic prospective observational study to derive and validate a clinica algorithm for the diagnosis of urinary tract infection in children presenting to primary care with an acute i. Health Technol. Assess. 20, 1-294 (2016).

40. Pamp, S. J., Gjermansen, M., Johansen, H. K. \& Tolker-Nielsen, T. Tolerance to the antimicrobial peptide colistin in Pseudomonas aeruginosa biofilms is linked to metabolically active cells, and depends on the pmr and mexAB-oprM genes. Mol. Microbiol. 68, 223-240 (2008).

41. Kim, J., Pitts, B., Stewart, P. S., Camper, A. \& Yoon, J. Comparison of the antimicrobial effects of chlorine, silver ion, and tobramycin on biofilm. Antimicrob. Agents Chemother. 52, 1446-1453 (2008).

42. Herrmann, G. et al. Colistin-Tobramycin combinations are superior to monotherapy concerning the killing of biofilm Pseudomonas aeruginosa. J. Infect. Dis. 202, 1585-1592 (2010).

43. Bjarnsholt, T. et al. Pseudomonas aeruginosa tolerance to tobramycin, hydrogen peroxide and polymorphonuclear leukocytes is quorum-sensing dependent Microbiology 151, 373-383 (2005).

44. Hu, Y. et al. Combinatory antibiotic therapy increases rate of bacterial kill but not final outcome in a novel mouse model of Staphylococcus aureus spinal implant infection. PLOS ONE 12, e0173019 (2017).

45. Hengzhuang, W. et al. High $\beta$-lactamase levels change the pharmacodynamics of $\beta$-lactam antibiotics in Pseudomonas aeruginosa biofilms. Antimicrob. Agents Chemother. 57, 196-204 (2013).

46. Marcus, R. J. et al. Biofilms in nephrology. Expert. Opin. Biol. Ther. 8, 1159-1166 (2008).

47. Klingstedt, T. et al. Synthesis of a library of oligothiophenes and their utilization as fluorescent ligands for spectral assignment of protein aggregates. Org. Biomol. Chem. 9, 8356-8370 (2011).

48. Sigurdson, C. J. et al. Novel pentameric thiophene derivatives for in vitro and in vivo optical imaging of a plethora of protein aggregates in cerebral amyloidoses. ACS Chem. Biol. 4, 673-684 (2009).

49. Åslund, A. et al. Studies of luminescent conjugated polythiophene derivatives: enhanced spectral discrimination of protein conformational states. Bioconjug. Chem. 18, 1860-1868 (2007).

(i) Open Access This article is licensed under a Creative Commons Attribution 4.0 International License, which permits use, sharing, adaptation, distribution and reproduction in any medium or format, as long as you give appropriate credit to the original author(s) and the source, provide a link to the Creative Commons license, and indicate if changes were made. The images or other third party material in this article are included in the article's Creative Commons license, unless indicated otherwise in a credit line to the material. If material is not included in the article's Creative Commons license and your intended use is not permitted by statutory regulation or exceeds the permitted use, you will need to obtain permission directly from the copyright holder. To view a copy of this license, visit http://creativecommons. org/licenses/by/4.0/.

(c) The Author(s) 2018 